\title{
L'irruption du numérique au Sud : le cas de
} l'Afrique

Digital technology incursion in the South: African case

La irrupción del sistema digital en el Sur: el caso de Àfrica

\section{Alain Bienaymé}

\section{CpenEdition}

Édition électronique

URL : http://journals.openedition.org/ctd/293

DOI : $10.4000 /$ ctd. 293

ISSN : 2491-1437

Éditeur

Chaire Unesco Pratiques émergentes en technologies et communication pour le développement

Référence électronique

Alain Bienaymé, "L'irruption du numérique au Sud : le cas de l'Afrique », Communication, technologies et développement [En ligne], 5 | 2018, mis en ligne le 02 janvier 2018, consulté le 10 décembre 2020.

URL : http://journals.openedition.org/ctd/293 ; DOI : https://doi.org/10.4000/ctd.293

Ce document a été généré automatiquement le 10 décembre 2020.

Communication, technologies et développement 


\title{
L'irruption du numérique au Sud : le cas de l'Afrique
}

\author{
Digital technology incursion in the South: African case \\ La irrupción del sistema digital en el Sur: el caso de Àfrica
}

\section{Alain Bienaymé}

\section{NOTE DE L'AUTEUR}

\section{INDONÉSIEN}

Abstrak: Jika era digital dimulai di negara- negara Utara melalui komputer dan telekomunikasi, maka di negara- negara Afrika era dgital dilakukan melalui penggunaan smartphone. Alat ini banyak digunakan, terutama oleh generasi muda. Peningkatan interkoneksi ini diharapkan dapat mempercepat proses tinggal landas menuju negara yang lebih mandiri dan maju.

Keterbelakangan ekonomi akibat sistem produksi diharapkan dapat dikurangi melalui kegiatan diversifikasi, yang dipusatkan pada pemenuhan kebutuhan lokal (pertanian dan perikanan, perdagangan, transportasi, layanan pos dan keuangan, kesehatan dan pendidikan). Perangkat digital berkontribusi mengumpulkan diaspora Afrika yang diharapkan dapat membantu menyampaikan pengalaman mereka kepada orang-orang dinegara asalnya. Namun agar negara berhasil lepas landas, masyarakat di Afrika harus dapat membuktikan melalui penciptaan pekerjaan yang bersih, tidak hanya di bidang telekomunikasi namun juga dapat dimulai dalam produksi barang dan jasanya secara digitalisasi. Agar berhasil Negara-negara di Afrika harus mempunyai metode yang dapat digunakan untuk mengatasi masalah tersebut, sehingga hambatan yang ada dapat diselesaikan dan hasilnya bisa digunakan untuk mendapatkan keuntungan.

Kata kunci: digital, selatan, Afrika, pembangunan, telepon genggam

1 L'Afrique est-elle enfin bien partie ? Une interrogation récurrente, lancinante à laquelle René Dumont répondit il y a 50 ans en limitant sa réponse à l'Afrique intertropicale. La question se pose aujourd'hui en des termes nouveaux : deux générations se sont succédé 
depuis que l'ensemble des nations du continent ont conquis leur indépendance. L'histoire justifie que l'on adresse la question à l'Afrique tout entière. On doit certes se garder de prétendre la considérer comme un tout homogène. Cette région du monde comprend en effet avec la République sud-africaine un pays qui s'est introduit dans le club très fermé des "grandes" économies émergentes initialement dénommé BRIC. Elle englobe également outre les nations du Maghreb, la Libye et l'Égypte et regroupe au total 54 États ${ }^{1}$ . La diversité des climats et des peuples, celle des situations économiques et politiques ne doivent pas masquer leurs traits communs. Et l'échelle du continent s'impose d'autant plus que la révolution industrielle des moyens de communication traverse les océans et survole les frontières qui délimitent continents et nations. Ainsi, en dépit et au-delà de leurs différences, les nations du Sud de tous les continents, mieux à même de confronter leurs atouts et leurs besoins respectifs, se découvrent des affinités que les nouveaux moyens de communication permettent d'exploiter au service de leur développement. Certains pays d'Afrique entretiennent, par exemple avec le Brésil, l'Inde ou la Chine, des rapports qui vont au-delà des seuls échanges de marchandises et concernent des accords de coopération portant sur des projets précis. Ces transferts d'expériences ponctuelles entre acteurs de terrain disséminés sur de vastes espaces pouvaient difficilement se produire naguère lorsque trois agences de presse occidentales, la TSF, le courrier postal et le téléphone fixe contrôlaient la transmission des informations à longue distance.

2 L'économie mondiale s'est engagée, sous l'impulsion du Nord, depuis une vingtaine d'années, dans une aventure industrielle d'envergure planétaire. Qu'on l'appelle révolution, basculement ou transition numérique, l'ensemble des phénomènes liés à l'essor d'Internet et des TIC modifie, entre autres, assez radicalement l'organisation des marchés, des entreprises et des processus de production, sans même parler de la vie sociale et culturelle ${ }^{2}$. Pour autant, l'expression de «néocapitalisme » employée pour qualifier les changements opérés dans les économies avancées est inappropriée dans le cas de l'Afrique ; en ce qui la concerne, on manifeste plutôt une certaine curiosité pour la naissance de ce que certains qualifient "d'afro-capitalisme». Et si l'infrastructure matérielle des TIC (câbles, satellites, équipements électroniques) est globale, si les pratiques digitales se ressemblent, en revanche les usages, les applications, les contenus informationnels portent la marque des caractéristiques nationales et locales ${ }^{3}$. On ne peut évaluer les apports du numérique en faisant abstraction des sociétés dans lesquelles il s'insère, des pratiques qu'il influence, de son environnement culturel. Or, l'Afrique est une partie du Sud de la planète qui présente bien des particularités qu'on ne retrouve pas, ou à moindre degré ailleurs, tant au Nord qu'en Asie et en Amérique latine. Ces particularités, notamment linguistiques, incitent à parler plus volontiers du numérique comme d'un outil sans passé industriel local plutôt que d'une véritable révolution ${ }^{4}$.

\section{Le contexte démo-économique}

3 Dans notre monde vieillissant, l'Afrique abrite une population jeune; les habitants de moins de 15 ans y représentent $41 \%$ de la population totale en comparaison d'une moyenne mondiale de $25 \%$. Selon les projections de l'UNICEF, $41 \%$ des naissances du monde se produiront en Afrique en $2050^{5}$. Les régions du continent sont inégalement avancées dans leur transition démographique. L'Afrique australe précède les autres avec un taux de fécondité modeste de 2,5 enfants par femme en âge de procréer, suivie de l'Afrique méditerranéenne (3,2), contre 5 à 6 pour l'Afrique subsaharienne ${ }^{6}$. 
4 La transition démographique ouvre une période favorable à l'accélération de la croissance économique. En effet la population active des 15 à 64 ans croit plus vite que celle des enfants de 0 à 14 ans et celle des personnes âgées ${ }^{7}$. Les jeunes générations particulièrement réceptives à l'égard des convivialités digitales sont un atout sur lequel l'Afrique devrait pouvoir compter pour stimuler son développement. Le continent pourrait relayer la Chine des années 1970-2000 et bénéficier à son tour de ce que les économistes appellent le dividende démographique pendant la période au cours laquelle le rythme des naissances ralentit alors que le poids des populations de retraités reste modeste, pour un temps...

Mais, à la différence de la Chine, l'Afrique est une constellation d'États jaloux d'affirmer leur souveraineté. Quelles que soient les disparités des conditions de vie entre les provinces chinoises, les autorités politiques de Pékin se doivent de maintenir la cohésion de l'ensemble et d'assurer un minimum de solidarité nationale par des réformes appropriées. En revanche, les outils numériques ne changent rien au fait qu'ils pénètrent un continent africain rassemblant des nations indépendantes dont les niveaux de PIB par tête sont très inégaux : en dollars courants leur PIB par habitant s'étalait en 2014 entre 286 dollars (Burundi) et un peu plus de 10000 dollars (Gabon et Maurice). Le Maghreb occupe une place intermédiaire : le PIB par habitant de l'Algérie est de 5498 dollars, celui du Maroc de 3103 dollars, et celui de la Tunisie de 4316 dollars. En comparaison, l'éventail des PIB par tête est beaucoup plus resserré en Europe de l'Ouest ; ils varient seulement de 30262 dollars (Espagne) à 60634 dollars (Danemark). Seuls la Norvège et Luxembourg dépassent les 100000 dollars 8 .

6 Les populations concernées sont encore en majorité rurales. Mais l'urbanisation accélérée soumet les grandes villes d'Afrique à la pression incontrôlée d'une multitude de jeunes gens à la recherche d'emplois mieux rémunérés. L'inégalité des conditions de vie à l'intérieur de chaque pays est considérable, y compris dans les mégapoles en forte expansion. La plupart des habitants éprouvent encore du mal à s'extirper de la pauvreté et souffrent de trois carences : l'alimentation en eau salubre, l'accès à l'électricité, les réseaux de transport délabrés.

7 D'aussi faibles ressources concernant des biens communs essentiels retentissent sur les niveaux de la productivité du travail et plus largement la productivité de l'ensemble des facteurs production, celle qui tient compte du gaspillage de capital résultant du mauvais usage et de la dégradation des matériels d'équipement. Les techniques de production agricole et artisanale sont encore rudimentaires ; le secteur informel résiste et l'appareil productif se limite pour l'essentiel aux industries extractives et aux cultures exportables. Par conséquent, les économies exportatrices de matières premières quasi brutes dépendent des marchés étrangers et subissent les aléas de la conjoncture internationale. Si elles ont bénéficié d'une croissance mondiale soutenue dans les années 2000-2010, elles pâtissent plus que les autres du ralentissement de la période actuelle et de la chute des cours des matières premières. Ainsi l'Algérie, comme en Amérique latine le Venezuela, est gravement affectée par la baisse de plus de moitié du prix du pétrole intervenue en 2015 ; l'état de l'économie de ces pays est révélateur de l'incapacité de leurs gouvernants à tirer parti de la rente pétrolière dégagée dans les années fastes pour diversifier leurs appareils de production. Le numérique pourrait changer les ressorts de la croissance future du continent.

8 À quels signes se manifeste l'irruption du numérique en Afrique ? Si dans les économies du Nord, le numérique a progressé avec Internet (depuis 1994) dans le sillage des 
ordinateurs et de l'informatique, au Sud le téléphone mobile, relativement peu onéreux, s'est imposé d'emblée comme la porte d'entrée du monde digital. En Afrique on comptait, en 2014, 851 millions d'abonnés, soit $72 \%$ de la population (contre $1 \%$ en 2000). Compte tenu de la possession de plusieurs téléphones par personne, on comptait 400 millions d'utilisateurs uniques à la même époque. Le smartphone est devenu en peu d'années le premier moyen de diffusion de l'information, loin devant la presse, et le nombre de ses abonnés devance celui des internautes qui approche les 200 millions. Les taux d'accès varient sensiblement d'un pays à l'autre. Mais ils sont tous en forte progression au point que les entreprises fournisseuses des matériels et des services des TIC tels que Orange, Google, entendent bâtir leur avenir en s'appuyant sur le dynamisme des marchés africains ${ }^{9}$. Dans ces conditions très rapidement changeantes, l'outil numérique annoncet-il un modèle de développement approprié ? Serait-il capable de mettre enfin l'Afrique sur la bonne voie?

\section{L'outil numérique, probable accélérateur du développement}

9 Pour en juger, on commencera par avancer l'hypothèse que l'élévation à un niveau d'interconnexion sans précédent des populations intéressées fait du numérique un probable accélérateur de développement en Afrique. On invoquera trois raisons pour étayer cette hypothèse.

10 La première se réfère à ce que Lionel Zinsou désigne les " avantages de l'arriération ${ }^{10}$ ». L'actuel premier ministre du Bénin, normalien, ancien cadre de l'entreprise multinationale BSN, puis dirigeant du fonds d'investissement PAI, recourt à cette formule pour évoquer le fait que les "modèles de l'économie numérique ne détruisent pas l'économie telle qu'elle était organisée auparavant ${ }^{11} »$. Le passé technologique et gestionnaire des entreprises quasi-inexistant ne dresse pas d'obstacle majeur à des projets économiques concurrents qui viendraient de la base. On détaillera quelques-unes des nombreuses initiatives qui sont engagées dans un nombre appréciable de pays du continent. Elles ne portent pas ombrage aux rares industries de transformation existantes. Dans une première étape, les téléphones portables peuvent au contraire stimuler les activités locales, ne serait-ce qu'en informant les artisans et petits producteurs sur les conditions du marché, en facilitant les échanges d'expériences et le règlement des échanges.

11 La deuxième raison tient à ce qu'il n'y a pas de développement possible sans échanges ni d'échanges sans communications. L'historien Pierre Chaunu ${ }^{12}$ attribue l'origine des processus de développement au rôle déterminant que l'imprimé, le langage écrit, les livres ont pu, en leur temps, au XVIII ${ }^{\mathrm{e}}$ siècle, jouer comme moteur du décollage de l'Europe de l'Ouest. Or, malgré les différences de forme entre tel ouvrage ou article et le moindre SMS, les uns et les autres diffusent des informations, lancent des idées neuves qui font rapidement leur chemin ${ }^{13}$. Le portable, puis l'iPhone de poche, les tablettes et l'ordinateur touchent directement et largement de jeunes générations, séduites par la simplicité des instruments et désireuses de s'en servir pour entreprendre et améliorer leur sort. Les individus se libèrent des pesanteurs ancestrales, conquièrent leur autonomie et approchent aisément d'autres interlocuteurs que les compagnies minières et pétrolières et les fonctionnaires de l'administration centrale. Avec le numérique, 
l'information interactive facilite les contacts multilatéraux, intragénérationnels et non plus seulement ceux qui empruntent la voie classique descendante et ascendante.

Soulignons enfin ce que peuvent apporter les diasporas africaines, le retour au pays de professionnels qualifiés soucieux de transmettre leur expérience, d'attirer des compatriotes aujourd'hui diplômés et pour certains devenus professeurs dans les universités occidentales. Le numérique entretient cette dynamique de rapprochement entre peuples de même origine que l'on voit éclore; ses canaux ouvrent de nouveaux horizons, désenclavent les esprits comme les territoires, étendent les marchés, encouragent l'esprit d'entreprise. Quand on observe l'état du système scolaire, la misère des universités publiques, la décrépitude de leurs bibliothèques, l'outil numérique dégage des perspectives qui aideront à combler ces lacunes. L'organisation et la gestion même de ces établissements, et notamment ceux du Cameroun, que nous avons analysés, peuvent tirer profit de l'utilisation des instruments numériques, ne serait-ce qu'en rendant leur gestion plus transparente sur trois actions essentielles : l'information sur les formations, le suivi des étudiants, les conditions de délivrance des diplômes ${ }^{14}$. Ce qui devrait être complété par d'autres initiatives dont il est maintenant question.

\section{Où en est-on aujourd'hui sur le plan des réalisations ?}

13 Walt Whitman Rostow ${ }^{15}$, qui avait anticipé l'avènement des économies émergentes, avança, avec son concept de décollage (take off), l'hypothèse que pour parvenir au stade de la croissance auto-entretenue, le pays doit avoir au préalable suffisamment investi et pendant assez longtemps, pour élever son niveau technique et sa productivité. Il faut pour cela que l'étendue du marché permette d'exploiter les économies d'échelle, ce que l'étroitesse traditionnelle de la demande intérieure interdit. Le numérique peut agir favorablement dans toutes ces directions.

14 D'une enquête touchant les sites affichés sur la toile et des conclusions dégagées dans de récents colloques consacrés à l'avenir de l'Afrique, on retire l'impression qu'un nombre appréciable de pays sont à l'aube et sur la piste d'un véritable décollage économique. La preuve devra certes en être donnée sur le plan de la création nette d'emplois. Ceux-ci ne touchent pas seulement les télécommunications qui sont les premières concernées. Mais - ce qui est plus convaincant - la création d'emplois doit intéresser tous les autres secteurs d'activité utilisateurs des infrastructures numériques et à laquelle le développement doit logiquement, sinon spontanément, amener.

L'économie numérique concerne en effet trois catégories d'emplois : la construction des infrastructures de réseaux (fibres, satellites, appareils téléphoniques...), les métiers périphériques (conceptions de logiciels, d'applications, de plateformes d'échanges, vidéos, services culturels...), et l'ensemble des autres secteurs dont les entreprises tirent profit d'un usage intense des TIC. Les investissements consacrés à la production des matériels d'infrastructure déclenchent ainsi un double effet multiplicateur d'emplois qui touche de proche en proche les entreprises de la périphérie, puis le reste de l'économie.

Pour que cet effet multiplicateur d'emplois se produise, il faut que la qualification des entrepreneurs et la formation technique des personnels répondent aux attentes suscitées par l'émergence de nouveaux métiers. Ce qui n'exclut pas dans un premier temps le rôle de la débrouillardise. Mais ce qui suppose surtout une étroite coopération des entreprises leaders avec les initiatives de terrain. Les sociétés multinationales telles qu'Orange et 
Google voient dans les marchés africains des relais de croissance significatifs. Orange tire d'ores et déjà $11 \%$ de son chiffre d'affaires d'Afrique. En 2008, Tidjane Dème a été nommé directeur de Google pour l'Afrique francophone. Cet ingénieur et polytechnicien sénégalais ambitionne de créer des produits de contenu pertinent pour l'Afrique. De même, un réseau anglophone Alliance for an Affordable Internet (A4AI) coordonne aujourd'hui les efforts d'environ 70 partenaires dont la World Web Foundation, des États comme le Nigeria, des firmes de télécoms (Ericsson, Alcatel-Lucent), mais aussi Google, Intel, Cisco, Microsoft...

Il n'existe certes aucun décompte systématique des entreprises qui se sont lancées dans l'univers du numérique à l'échelle du continent. Toutefois, parmi les initiatives les plus dignes d'intérêt, on soulignera l'importance prise par le i-hub de Nairobi qui affirme son ambition de devenir la (une ?) Silicon Valley de l'Afrique ${ }^{16}$. Ce centre très actif est né en 2007; il abrite en 2015 plus d'une cinquantaine de jeunes pousses versées dans les technologies de pointe. Leur objectif est de concevoir des logiciels diffusables dans tout le continent : remplacement de l'éclairage urbain en lampes à kérosène par des lampes à énergie solaire payée à l'heure via des applications SMS, livraison de colis par motos équipées de GPS, offre d'adressage postal à des Kenyans sans adresse, règlement des transactions financières par appareils portables très répandu au Kenya... D'autres espaces d'incubation ouvrent leurs portes, par exemple au Ghana. Le multiplicateur d'emplois a d'autant plus de chances d'être élevé que les économies réceptrices sont déjà diversifiées, ce qui est le cas en Afrique du Sud, Botswana, Éthiopie, Rwanda, Côte d'Ivoire entre autres. Les innovations portent la marque du génie propre des habitants de ces pays. On signale par exemple au Togo une entreprise qui recycle les rebuts de matériel informatique pour les convertir en imprimantes 3D. Des projets analogues concernent également la gestion des transports et des déchets dans les mégapoles, la conception écologique des villes africaines de demain. Ainsi, on comptait, en 2013, 52 villes de plus d'un million d'habitants ; leur nombre doublera d'ici 2030.

Un des domaines de prédilection du numérique est le commerce en ligne qui a vocation à compléter un négoce archaïque. Ainsi Junia a été fondée en 2007 par un Ghanéen de 29 ans de retour des États-Unis et un Nigérian de 36 ans; leur plateforme numérique emploie 450 personnes (2013) et vend 50000 produits. Elle entend devenir une Amazon Afrique en conquérant les marchés francophones. Rocket Internet, la société allemande qui a financé le départ de cette start-up est un incubateur qui revendique la création de 100 entreprises dans 40 pays avec un effectif total de 15000 employés.

Plusieurs services tireront les plus grands bienfaits du numérique ; et au premier plan des services publics: l'éducation, la finance, la santé. Par exemple, Thierry N'Doufou, l'inventeur ivoirien de la première tablette éducative africaine en 2014, a mis au point, après un premier succès, la deuxième version de la tablette Qelasy. Ce pionnier espère la faire adopter par tous les établissements scolaires primaires de son pays d'origine et audelà. Les établissements d'enseignement qui peinent à renouveler leur documentation recevront ainsi sans retard les nouveautés publiées au Nord. De futures extensions au bénéfice de l'enseignement secondaire et universitaire et de l'apprentissage sont à prévoir. Pour qui connaît l'état de délabrement dans lequel se trouvent les bibliothèques scolaires et universitaires, le numérique devrait apporter une aide inespérée. Par exemple Facebook, EDX et le gouvernement du Rwanda unissent leurs efforts pour faciliter l'accès à la plateforme Harvard-MIT et dispenser des cours en ligne. En ce qui concerne la santé et pour pallier le faible nombre des maternités, Lionel Zinsou envisage de recourir à 
l'échographie à distance. Le numérique offre l'occasion historique de réinventer les services de santé en créant des applications adaptées. Et s'agissant des activités financières, les carences de l'administration postale peuvent être contournées par le développement des factures sur portable.

Les services privés voient monter en puissance l'usage de l'outil numérique: Kampala, capitale de l'Ouganda, tire argument de la vitesse de connexion pour vanter les cybercafés de ses hôtels. On mentionnera aussi la société Skopeo créée en 2012 par un FrancoGuinéen, ingénieur de l'École des Mines et MBA de HEC ; c'est un incubateur de solutions mobiles et de traitement et d'exploitation des données destiné (cloud computing) à offrir de multiples applications aux entreprises et aux particuliers : géolocalisation pour taxis, suivi des approvisionnements des entreprises de distribution commerciale, gestion des activités des enfants, gestion d'inventaires. Nombre de progiciels ajustés au plus près des besoins des professions devraient voir le jour et faciliter l'éclosion de nouvelles entreprises ${ }^{17}$.

\section{Des freins à desserrer}

21 À l'image d'une constellation qui, d'année en année s'emplirait d'étoiles nouvelles, les initiatives se multiplient sur le continent africain grâce à l'outil numérique ${ }^{18}$. Toutefois, les premiers succès ne doivent pas faire oublier les obstacles qui empêchent d'exploiter au mieux les potentialités de l'outil. Quels sont ces obstacles?

Tout d'abord, les deux tiers de la population du continent restent privés d'électricité; pour eux, la bougie, le charbon, le bois et l'essence offrent des substituts coûteux et de qualité médiocre. Le taux d'accès de la population à l'électricité est selon l'Agence Internationale de l'Énergie de $30 \%$ en Afrique subsaharienne. Il devrait atteindre les $66 \%$ en 2030. Il reste très inégalement réparti: $9 \%$ des citoyens de la République centrafricaine et $25 \%$ des Kenyans seulement en bénéficient. Ce handicap retarde l'accès à Internet et oblige les abonnés du téléphone à se déplacer pour recharger leurs mobiles dans les cybercafés les plus proches. Le coût du kilowattheure produit par les générateurs à pétrole peut atteindre jusqu'à 10 dollars par comparaison avec le coût du kilowattheure de 0,135 dollar à Paris. On évalue à 250 milliards de dollars les investissements nécessaires pour électrifier tout le continent à partir des énergies fossiles et renouvelables. La Banque Africaine de Développement s'est engagée dans deux projets au Maroc et au Kenya concernant le solaire et la géothermie. L'Europe ne reste pas indifférente dans la recherche de solutions; Jean-Louis Borloo en éloquent avocat de cette grande cause, s'emploie activement à faire avancer le vaste chantier qu'est l'électrification du continent dont les infrastructures devront être construites, puis exploitées et entretenues avec compétence.

23 L'accès à Internet haut débit pose en effet des problèmes de capacité et de connectivité. En 1994, seules l'Afrique du Sud et l'Égypte étaient connectées à la Toile. D'ores et déjà, 16 câbles sous- marins relient l'Afrique au reste du monde et presque toutes les nations africaines sont reliées au réseau. Mais la construction des réseaux de distribution est onéreuse ; la faible rentabilité des derniers kilomètres de fibre optique requis pour relier les entreprises et les foyers disséminés sur tout le territoire retarde les investissements de connexion. Or, la densité de la population du continent, quoiqu'en hausse, reste quatre fois plus faible que dans l'U.E. Elle est de 40 habitants au kilomètre carré, contre 390 en Inde. Les populations côtières et urbaines sont dans ces conditions les premières servies, 
ce qui ne manque pas d'entretenir les frustrations locales et d'encourager l'émigration vers les villes.

En outre, nombre d'innovations isolées ne porteront leurs fruits que si elles appellent des investissements complémentaires. Les projets de transport par drones d'échantillons de sang destinés à des populations éloignées sont de faible utilité si le manque de routes empêche les médecins de visiter leurs patients ${ }^{19}$.

Ces obstacles naturels s'accompagnent souvent d'une réglementation archaïque et paralysante. Son évolution est tributaire d'un contexte politique souvent ambigu. Les experts de la Banque mondiale qui se sont interrogés récemment sur l'avenir de l'Afrique se sont efforcés de départager deux points de vue contradictoires ${ }^{20}$. Il ne faut pas s'en étonner : l'avenir de tout pays en développement inspire en général des thèses opposées. $\mathrm{Au}$ chapitre du pessimisme et notamment dans le cas africain, on s'inquiète d'abord et à juste titre de la dépendance persistante de nombreux pays à l'égard des industries exportatrices de matières premières; une dépendance qui souligne la faiblesse de la contribution des activités manufacturières au PIB. De plus, l'instabilité politique alimente des guerres civiles dans environ un tiers des États de l'Afrique subsaharienne et qui n'épargne pas les pays riverains de la Méditerranée. Ajoutons que les inégalités de revenus reflètent l'accaparement des richesses naturelles par des minorités; leur détournement s'opère au détriment des investissements productifs. Enfin la corruption rampante, que certains politologues qualifient d'exercice de micro-politique par les détenteurs de la moindre parcelle de pouvoir se fait aux dépens de l'efficacité des services publics de l'éducation, de la santé et de la construction. Dans un tel contexte, l'avènement d'Internet peut être vécu comme une menace par tous les potentats, petits ou grands, en mettant au grand jour leurs pratiques dans l'exercice du pouvoir et la protection de leurs rentes.

Plus généralement, la progression du numérique propage dans les sociétés de cultures différentes des nouvelles habitudes de vie et de travail qui se heurtent à des réticences. Le numérique dérange et modifie le rapport au temps des acteurs ; les impératifs d'efficacité, de productivité, de rentabilité dont s'accommodent les sociétés du Nord doivent composer avec d'autres dimensions de la culture locale, du moins si l'on admet l'hypothèse que la culture se réfère à trois composantes distinctes : la mémoire vivante de la beauté, l'efficacité et le professionnalisme, mais aussi le bien-vivre ensemble autour de pratiques ancestrales (parole, croyances, coutumes religieuses, vestimentaires, alimentaires... $)^{21}$.

Au chapitre des raisons d'espérer, nos experts précités de la Banque mondiale s'appuyant sur l'importance aujourd'hui reconnue du renforcement du capital humain, soulignent la contribution que les TIC, entre autres, doivent pouvoir apporter pour faire progresser la santé et l'éducation des populations. Et nos experts de conclure que «les deux récits réducteurs n'empêchent pas le point de vue des optimistes d'être en dernière instance plus près de la réalité2 ${ }^{22}$ ». En effet, un certain nombre de pays ont amorcé leur transition démocratique à la suite du Bénin dont le Gouvernement fut un des premiers à ouvrir un large débat avec la société civile en 1990. La promotion de la société civile est un résultat non négligeable de l'émancipation intellectuelle des individus et la diffusion des informations par les instruments numériques doit donner les moyens de lutter contre toute forme d'obscurantisme et d'abus de pouvoir.

Il reste que l'accueil réservé par les différents pays du continent aux innovations induites par les instruments numériques demeure très inégal même entre pays voisins. Sans 
même évoquer la possible réticence de certains gouvernements à l'égard d'un système d'informations planétaire difficile à contrôler, les administrations tardent à s'adapter. Au Maghreb, la proportion d'internautes varie entre $56 \%$ au Maroc, $44 \%$ en Tunisie et $28 \%$ en Algérie ; ce dernier pays est classé 163e sur 184 en ce domaine. Des États pauvres ou mal gouvernés et inattentifs aux aspirations des jeunes générationscoexistentavecd'autresdontl'appareilpolitiqueestdavantageenphaseavecl'économie émergente. En septembre 2015, le monde a été informé que la Côte d'Ivoire entend accélérer le déploiement de la fibre optique, que le Sénégal a créé un laboratoire de pointe en cybersécurité, que l'ARPT algérienne va interconnecter téléphones fixes et portables et que la Tunisie renforce sa vigilance sur les réseaux sociaux. Tout ne relève certes pas de l'État. Comme il a été souligné lors d'un forum récemment tenu à Abidjan, de bons maires sont indispensables à la réussite de tels projets, tel Urban Futures, nourri par la fondation suédoise Mistra; et de citer comme exemples de bonne gestion les villes de Lagos et Windhoek. L'association française "Villes Internet »"23, créée en 1999, coordonne le développement et l'animation d'un réseau des «acteurs de l'internet citoyen", et tout particulièrement des élus et agents des collectivités qui animent le territoire public français. Son expérience peut profiter aux municipalités africaines qui le souhaiteraient.

Certes, les statistiques ne permettent pas de mesurer l'ampleur des vrais succès ni de les distinguer des effets d'annonce. Mais, au total, le sentiment domine que le numérique place une bonne partie du continent au début d'un véritable décollage économique. Internet peut aider les gouvernements à moderniser leurs pratiques et accélérer les coopérations transfrontières. Il faut ici mentionner ici le partenariat Inde-Afrique qui vient d'être conclu: il prévoit l'installation d'un réseau de télécommunications reliant l'Inde à 42 États africains. Ce réseau fournira des services de télémédecine et un accès à l'enseignement à distance. Le secteur privé peut contribuer activement à ces coopérations. Ainsi, en 2012, au Maroc, un ancien contrôleur financier du groupe pharmaceutique Sanofi et ancien cadre de sociétés allemandes et françaises a fondé à Casablanca une plateforme de e-recrutement et de recherche d'emplois sur Internet dénommée Africawork. Pour le moment, avec une trentaine de collaborateurs, Africawork est présente dans 12 États francophones et dans 5000 sociétés $^{24}$.

\section{Conclusion}

L'investissement dans les TIC dans les économies du continent africain est devenu un sujet majeur d'actualité, et, à défaut d'un inventaire systématique des initiatives et de leurs réalisations, de nombreux jeunes acteurs s'emparent de l'outil numérique pour en faire le moteur d'une véritable diversification du tissu économique. Parvenu au terme de cet essai, le lecteur s'étonnera de la différence de tonalité dans l'accueil réservé à l'économie numérique au nord et au sud de la planète. L'enthousiasme des innovateurs et des incubateurs aux États-Unis et que partage l'Europe du Nord rencontre plus de réticence dans les milieux intellectuels et en Europe méridionale. En effet, l'économie numérique est d'abord jugée à l'aune de la croissance et de l'emploi ; ce n'est pas tellement l'innovation en soi qui attire, mais le progrès que la société en retire concrètement $^{25}$. Le multiplicateur d'emplois c'est-à-dire le rapport entre le total des créations nettes d'emplois directement imputables à l'usage systématique de procédés numériques et les emplois spécifiquement affectés à la conception et à l'installation des 
outils numériques n'est pas aussi élevé qu'on s'y attendait. Les économies de maind'œuvre observées dans les services traditionnellement recruteurs de personnels s'ajoutent à celles qui résultent de la robotisation des industries manufacturières. La croissance du PIB s'en trouve freinée d'autant plus qu'une partie des activités lucratives entre particuliers échappe pour le moment aux estimations officielles. Les plateformes électroniques développent le secteur informel du Nord. La productivité par heure de travail progresse, mais le taux d'emploi de la population active stagne ou régresse avec le nombre des heures travaillées. Les trajectoires observées de 2010 à 2014 font état d'une augmentation du PIB par habitant en dollars courants de $13 \%$ aux États-Unis, $14 \%$ en Allemagne, $18 \%$ au Royaume-Uni, mais seulement de $5 \%$ en France et cet indicateur baisse de $-1,3 \%$ en Italie, $-1,5 \%$ en Espagne ${ }^{26}$.

Le Sud vit une situation différente. Le numérique y est considéré comme un progrès. Il crée des emplois. Il ouvre des perspectives sur un avenir meilleur; et le meilleur aux yeux des populations les plus pauvres n'a pas le même sens que pour les populations des pays riches. En effet, l'économie numérique offre l'occasion de diversifier les activités au-delà des industries extractives de matières premières et de répondre concrètement aux besoins du continent africain. Et ce sans provoquer de destructions d'emplois massives, sinon une diminution du secteur informel. De plus, l'usage des outils numériques ouvre la perspective de maitriser les maladies endémiques. Ce qui favorise et l'emploi et la productivité du travail. L'abaissement du coût des smartphones, la liberté d'accès à Internet, la formation des jeunes aux métiers du numérique (logiciels, construction de sites, applications, etc.) s'imposent donc comme une priorité de premier rang dans les stratégies de développement du Sud. En résumé le contraste entre le Nord et le Sud provient de ce que compte tenu de leurs histoires respectives, les peuples ont une perception différente du lien entre le progrès technique et le bien-être collectif. Il reste que la transition en cours multiplie les occasions de coopération intercontinentale dont le développement du continent africain doit pouvoir tirer profit.

\section{BIBLIOGRAPHIE}

Banque mondiale, «PIB (\$ US courants) », disponible sur donnees.banquemondiale.org.

Bauchard Claire, «Carte interactive : ces Français qui brillent en Afrique et en Europe », Les Dossiers de L'Opinion (Made Out of France), 16 octobre 2015, disponible sur http://www.lopinion.fr.

Bienaymé Alain, « Vers un plan stratégique de développement de l'enseignement supérieur du Cameroun », dans « Rapport sur le management de l'enseignement supérieur du Came- roun », rapport de la mission d'experts de l'UNESCO, mars-mai 1999.

Bordry Anne-Sophie, Bouverot Anne, « Internet mobile en Afrique : outil ou révolution? ", Problèmes économiques, $n^{\circ} 3102$ (Les Défis de l'Afrique), 2015, p. 38-43.

Delannoi Gilles ; « Qu'est-ce qu’une nation ? 1882-2015 », Commentaire, nº 154, été 2016.

Devarajan Shantayanan \& Fengler Wolfgang, “Africa's Economic Boom: Why the Pessimists and the Optimists are Both Right”, Foreign Affairs, may/june 2013. 
Dortier Jean-François, « Les gens aspirent au progrès, pas à l'innovation. Entretien avec Marc Giget », Problèmes économiques, $\mathrm{n}^{\circ} 3115$ (Les Technologies au cœur de la croissance), 2015, p. 16-20. Dumont René, L'Afrique Noire est mal partie, Le Seuil, coll. « Les Collections Esprit. Frontière ouverte ", Paris, 1962.

Fulda Anne, «Lionel Zinsou : "Le numérique, une chance pour l'Afrique” ", Le Figaro, 17 juillet 2015.

Google, site consulté : « Internet en Afrique ».

Le Monde Afrique, 9 septembre 2015.

Macharia Joel, «L'accès à Internet n'est plus un luxe », Afrique Renouveau, vol. 28, nº 1 ( Infrastructures : clés du progrès), 2014, p. 18-19, disponible sur http://www.un.org.

Martel Frédéric, Smart. Ces Internets qui nous rendent intelligents, Stock, Champs actuel, 2014.

Mataillet Dominique, « Plus de 4 milliards d'Africains en 2100 », Problèmes économiques, $\mathrm{n}^{\circ} 3102$ ( Les Défis de l'Afrique), 2015, p. 33-37.

Montbrial Thierry de, David Dominique (dir.), RAMSES 2015. Le défi des émergents, Dunod / Institut français des relations internationales, Paris, 2015.

Rostow Walt Whitman, The Stages of Economic Growth: A Non-Communist Manifesto, Cambridge University Press, Cambridge, 1960.

\section{NOTES}

1. Sur l'histoire du concept de nation voir Delannoi, 2016.

2. Sur la contribution des outils numériques à l'évolution culturelle des pays du sud de la Méditerranée, voir Bienaymé, 2015.

3. Martel, 2014.

4. Bordry \& Bouverot, 2015.

5. Mataillet, 2015.

6. Ainsi à la surprise de Ouadah-Bedidi \& al., la fécondité a rebondi en Algérie entre 2000 et 2010 de 2,1 à 2,9 (7,6 en1982) alors qu'elle s'est stabilisée au seuil de remplacement des générations au Maroc, en Tunisie et en Libye.

7. Devarajan \& Fengler, 2013.

8. Banque Mondiale, Montbrial David (dir.), 2015.

9. En 2014, la croissance estimée du PIB du continent était de 4,8 \% dont 5,8 \% pour l'Afrique subsaharienne. De 2010 à 2014 le PIB per capita a crû de 37 \% au Kenya, 25 \% en Côte d'Ivoire, 14 \% au Botswana, $11 \%$ au Maroc.

10. Fulda, 2015.

11. ibidem.

12. Chaunu, 1971.

13. Le nombre de langues et dialectes pratiqués en Afrique est estimé à environ 2000 ; une cinquantaine seulement sont parlées par plus d'un million de locuteurs.

14. Bienaymé, 1999.

15. Rostow, 1960.

16. Macharia, 2014.

17. Bauchard, 2015.

18. Le Monde Afrique, 9 septembre 2015. 
19. Ces projets intéressent le Rwanda, l'Ouganda, le Malawi : “Look before you leap", The Economist, 6/8/2016.

20. Devarajan, Fengler.

21. Devarajan, Fengler.

22. S. Devarayan \& W. fengler, art.cit.

23. http://www.villes-internet.net

24. Bauchard, 2015.

25. Dortier, 2015.

26. Banque mondiale.

\section{RÉSUMÉS}

Si le numérique a progressé au Nord avec Internet dans le sillage des ordinateurs et de l'informatique, au Sud le téléphone mobile, relativement peu onéreux, s'est imposé comme la porte d'entrée du monde digital. Il rencontre un large succès auprès des jeunes générations. L'élévation à un niveau d'interconnexion sans précédent des populations africaines fait du numérique un probable accélérateur de développement. L'arriération des structures de production ne dresse pas un obstacle infranchissable à la diversification des activités pour peu que les facilités de communication soient employées à satisfaire les besoins locaux : agriculture et pêcheries, commerces alimentaires, transports, services postaux et financiers, éducation et santé. Le numérique aide à mobiliser les diasporas africaines afin qu'elles transmettent à leurs compatriotes leur expérience de professionnels qualifiés. Si certains pays sont à l'aube d'un véritable décollage économique, la preuve devra en être donnée en terme de création nette d'emplois, non seulement dans les télécommunications, mais dans tous les autres secteurs d'activité utilisateurs des infrastructures numériques et que le développement devra entraîner. Il est fait état des initiatives qui se multiplient sur le continent africain grâce à l'outil numérique. Toutefois, les premiers succès ne doivent pas faire oublier les obstacles qui empêchent d'exploiter au mieux les potentialités de l'outil. Si ces obstacles concernent notamment le coût d'accès à l'électricité, ils tiennent pour la plupart à des méthodes de gouvernance inadaptées aux exigences du développement.

If the digital era began in the Northern countries through heavy expenditures in computers and telecoms, it penetrates African countries largely through the smartphones. These tools are widely used, particularly by the young generations. The raising level of interconnectedness within the populations would hopefully accelerate their take - off. The economic backwardness of their production systems do not raise insuperable obstacles to the diversification of activities, if they are focused on the satisfaction of the local needs (agriculture and fisheries, groceries, transportation, postal and financial services, health and education. The digital tools contribute to summon up the African diasporas and can be of a great help for the professionnals who wish to transmit their experience to their countrymen. If some countries are taking-off, it has to be proven through a net job creation not only in the telecoms, but also in the production of goods and services which get the most of the digital tools. Illustrations are given of the swarming of start-ups in the field of digitalization. However in order to succeed in their uprise and to take avantage of the potentialties, the African countries have to overcome the obstacle of the cost of access to electricity and to bring remedy to its weak governance methods. 
INDEX

Mots-clés : numérique, Sud, Afrique, développement, téléphonie mobile

Keywords : digital, South, Africa, development, mobile phones

\section{AUTEUR}

ALAIN BIENAYMÉ

Université Paris Dauphine 